\title{
Relativistic Descriptions of Final-State Interactions in Charged-Current Quasielastic Neutrino-Nucleus Scattering at MiniBooNE Kinematics
}

\author{
Andrea Meucci, ${ }^{1}$ M. B. Barbaro, ${ }^{2}$ J. A. Caballero, ${ }^{3}$ C. Giusti, ${ }^{1}$ and J. M. Udías ${ }^{4}$ \\ ${ }^{1}$ Dipartimento di Fisica Nucleare e Teorica, Università degli Studi di Pavia and INFN, Sezione di Pavia, \\ Via A. Bassi 6, I-27100 Pavia, Italy \\ ${ }^{2}$ Dipartimento di Fisica Teorica, Università di Torino and INFN, Sezione di Torino, Via P. Giuria 1, 10125 Torino, Italy \\ ${ }^{3}$ Departamento de Física Atómica, Molecular y Nuclear, Universidad de Sevilla, 41080 Sevilla, Spain \\ ${ }^{4}$ Grupo de Física Nuclear, Departamento de Física Atómica, Molecular y Nuclear, Universidad Complutense de Madrid, \\ CEI Moncloa, 28040 Madrid, Spain \\ (Received 26 July 2011; published 17 October 2011)
}

\begin{abstract}
The results of two relativistic models with different descriptions of the final-state interactions are compared with the MiniBooNE data of charged-current quasielastic cross sections. The relativistic mean field model uses the same potential for the bound and ejected nucleon wave functions. In the relativistic Green's function model, the final-state interactions are described in the inclusive scattering consistently with the exclusive scattering using the same complex optical potential. The relativistic Green's function results describe the experimental data for total cross sections without the need to modify the nucleon axial mass.
\end{abstract}

DOI: 10.1103/PhysRevLett.107.172501

PACS numbers: 25.30.Pt, 13.15.+g, 24.10.Jv

The double-differential cross sections for muon neutrino charged-current quasielastic (CCQE) scattering, recently measured by the MiniBooNE collaboration [1], have raised debate over the role of the various theoretical ingredients entering the description of the reaction. High-quality descriptions of the CCQE differential cross sections in the few-GeV region are required to support neutrino oscillation measurements $[1,2]$. The energy region explored requires a relativistic description of the process, where not only relativistic kinematics is considered but also nuclear dynamics and current operators should be described within a relativistic framework.

The simplest relativistic model to describe CCQE neutrino scattering is the relativistic Fermi gas (RFG). When a dipole shape is assumed for the axial form factor, the nucleon axial mass $M_{A}$ has been used as a free parameter within the RFG model. Indeed, the MiniBooNE cross section [1] is underestimated by the RFG unless $M_{A}$ is significantly enlarged $\left(1.35 \mathrm{GeV} / c^{2}\right)$ with respect to the accepted world average value $\left(1.03 \mathrm{GeV} / c^{2}\right.$ [3]). Thus, a larger axial mass within the RFG could be a way to effectively incorporate nuclear effects. More sophisticated models have been applied to neutrino-nucleus scattering. At the level of the impulse approximation (IA), models based on a realistic spectral function [4] or on the relativistic IA, which, contrarily to the RFG, are in good agreement with electron scattering data, also underestimate the experimental CCQE cross sections [5-7] unless $M_{A}$ is significantly enlarged.

It has been pointed out that, in some kinematic regions where the neutrino flux for the experiment has significant strength, the reaction may have sizable contributions from effects beyond the IA. The contribution of multinucleon excitations to CCQE scattering has been found sizable and able to bring the theory in agreement with the experimental MiniBooNE cross sections without the need to increase the value of $M_{A}[8,9]$.

Fully relativistic microscopic calculations of twoparticle-two-hole $(2 p-2 h)$ contributions are very involved and may be bound to model-dependent assumptions. Attempts have been made to incorporate more phenomenological knowledge in the predictions of these calculations. For instance, the part of the $2 p-2 h$ excitations which may be reached through two-body meson-exchange currents (MEC), in particular, the contribution of the vector MEC in the $2 p-2 h$ sector, evaluated in the model of Ref. [10], has been incorporated in a phenomenological approach based on the superscaling behavior of electron scattering data [6]. Although the inclusion of these $2 p-2 h$ MEC contributions yields somewhat better agreement with the data [7], the theory still lies below the data at larger angles.

Another essential ingredient of the reaction is the finalstate interactions (FSI) between the ejected nucleon and the residual nucleus. The relevance of FSI has been clearly stated for exclusive $\left(e, e^{\prime} N\right)$ processes, where the use of complex optical potentials in the distorted-wave impulse approximation is required [11-13]. In the analysis of inclusive reactions, FSI remain a crucial ingredient for a proper description of data [14-20]. All elastic and inelastic channels contribute to the inclusive process. Thus, the complex potential, with imaginary terms designed to reproduce just the elastic channel, should be dismissed. Different approaches have been used to account for FSI under inclusive conditions. For instance, in the approaches based on the relativistic distorted-wave impulse 
approximation, they have been accounted for by using purely real potentials. The final nucleon state has been evaluated with the real part of the relativistic energydependent optical potential (rROP) or with the same relativistic mean field (RMF) potential considered in describing the initial nucleon state $[18,19]$. However, the rROP is unsatisfactory from a theoretical point of view, since it is an energy-dependent potential, reflecting the different contribution of open inelastic channels for each energy, and, under such conditions, dispersion relations dictate that the potential should have a nonzero imaginary term [21]. On the other hand, the RMF model is based on the use of the same strong energy-independent real potential for both bound and scattering states. It fulfills the dispersion relations [21] and also the continuity equation. The RMF model applied to inclusive quasielastic (QE) $\left(e, e^{\prime}\right)$ processes describes scaling behavior and gives rise to a superscaling function with a significant asymmetry, in good agreement with data $[19,22]$.

A different description of FSI makes use of relativistic Green's function techniques [15,16,20,23]. Under suitable approximations [15,20,23-25], which are basically related to the IA, the components of the nuclear response are written in terms of the single-particle optical model Green's function. This result has been derived by arguments based on multiple scattering theory [21] or by means of projection operator techniques within nonrelativistic $[23,24]$ and relativistic $[15,20]$ frameworks. The formalism allows us to recover the contribution of nonelastic channels in the case of inclusive scattering, starting from the complex relativistic optical potential (ROP) which describes elastic nucleon-nucleus scattering data. The relativistic Green's function (RGF) model provides a consistent treatment of FSI in the exclusive and in the inclusive scattering and gives also a good description of $\left(e, e^{\prime}\right)$ data $[15,16]$. We note that, due to the analiticity properties of the optical potential, the Green's function model fulfills the Coulomb sum rule $[15,21,23,24]$.

The results of the RMF and RGF models have been compared for the inclusive QE electron scattering [16] and for the CCQE neutrino scattering [26]. Both models describe successfully the behavior of electron scattering data and their scaling and superscaling functions and both produce a significant asymmetry in the scaling function that is strongly supported by data. There are, however, some differences between the RMF and RGF results, depending on kinematics, which increase with the momentum transfer. Whereas the RMF may be considered as a faithful representation of the pure "nucleonic" contribution to the inclusive response, the RGF, on the contrary, may to some extent translate loss of elastic strength to non-nucleonic degrees of freedom, contributing to the imaginary optical potential, into inclusive strength predicted by the RGF.

In this Letter, the predictions of the RMF and RGF models are compared with the recent CCQE MiniBooNE data. The comparison between the results of the two models $[16,26]$ can be helpful for a deeper understanding of nuclear effects, more specifically FSI, which may play a crucial role in the analysis of CCQE data and its influence in studies of neutrino oscillations at intermediate to high energies. This is of particular interest for the case of the MiniBooNE CCQE data which, given the nature of the experiment, may receive important contributions from multinucleon as well as non-nucleonic excitations, which have to be estimated and removed from the CCQE data [27]. Thus, the RMF would represent a lower bound to MiniBooNE CCQE, while the RGF should yield larger predictions.

Details of the two models can be found in [18,19,22,28,29] for the RMF and in [15,20,23-26,30,31] for the RGF. In the RMF case, the components of the nuclear response are obtained from the sum over all the single-particle shell-model states of the squared absolute value of the transition matrix elements of the singlenucleon current. In the RGF case, the calculations require matrix elements involving the eigenfunctions of a complex optical potential and of its Hermitian conjugate $[15,20]$.

In both calculations, the bound nucleon states are selfconsistent Dirac-Hartree solutions derived within a RMF approach using a Lagrangian containing $\sigma, \omega$, and $\rho$ mesons [32]. The same real potential gives the scattering states in the RMF, whereas, in the RGF calculations, two parametrizations for the ROP have been used: the energy-dependent and $A$-dependent EDAD1 and the energy-dependent but $A$-independent EDAI-12C complex phenomenological potentials of [33], which are fitted to proton elastic scattering data on several nuclei in an energy range up to $1040 \mathrm{MeV}$. The comparison between the results obtained with two different phenomenological optical potentials may indicate how the incomplete determination of this important ingredient can influence the predictions of the model. In all the calculations, we have used the standard value of the nucleon axial mass, i.e., $M_{A}=1.03 \mathrm{GeV} / c^{2}$.

In Fig. 1, we show the CCQE double-differential ${ }^{12} \mathrm{C}\left(\nu_{\mu}, \mu^{-}\right)$cross section averaged over the neutrino flux as a function of the muon kinetic energy $T_{\mu}$. In each panel, the results have been averaged over the corresponding angular bin of $\cos \theta$, where $\theta$ is the scattering angle of the muon. The results evaluated with RMF and RGF with EDAD1 and EDAI potentials are compared with the MiniBooNE CCQE data [1].

The RMF results [7] yield reasonable agreement with data for small angles and low muon energies, the discrepancy becoming larger as $\theta$ and $T_{\mu}$ increase. The shape followed by the RMF cross sections fits well the slope shown by the data. A good agreement with the experimental shape is shown also by the RGF cross sections. The RMF and RGF models yield close predictions at larger values of $T_{\mu}$ for all the bins of $\cos \theta$ shown in Fig. 1. Notice, however, that the RGF cross sections are generally 


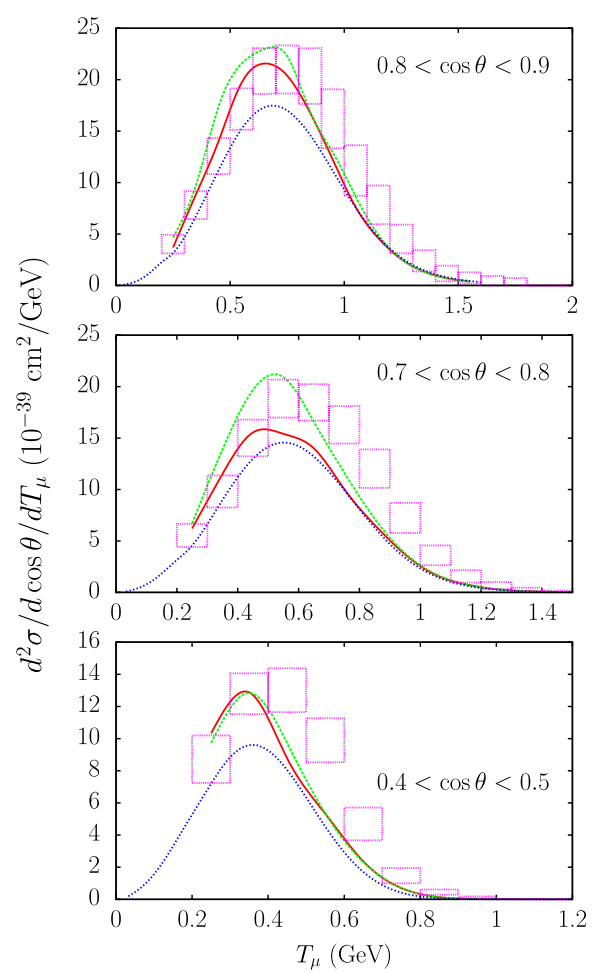

FIG. 1 (color online). Flux-averaged double-differential cross section per target nucleon for the $\mathrm{CCQE}{ }^{12} \mathrm{C}\left(\nu_{\mu}, \mu^{-}\right)$reaction calculated in the RMF [dotted dark gray (blue) line] and in the RGF with EDAD1 [solid (red) line] and EDAI [dotted light gray (green) line] potentials and displayed versus $T_{\mu}$ for various bins of $\cos \theta$. The data are from MiniBooNE [1]. The uncertainties do not include the overall normalization error $\delta N=10.7 \%$.

larger than the RMF ones, particularly around the peak region, where the RGF produces cross sections in reasonable agreement with data.

It is worth noticing that the differences between the RGF results obtained with the two optical potentials are enhanced in the peak region and are, in general, of the order of the experimental errors. The EDAD1 and EDAI potentials yield close predictions for the bin $0.4<\cos \theta<0.5$; small differences are seen in the bin $0.8<\cos \theta<0.9$, the RGF-EDAI cross section being larger than the RGFEDAD1 one, while the difference is sizeable for the bin $0.7<\cos \theta<0.8$, with the RGF-EDAD1 results closer to the RMF than to the RGF-EDAI ones.

The RMF model uses the effective mean field that reproduces the saturation behavior of nuclear matter and the properties of the ground state of nuclei. It includes only nucleonic contributions to the inclusive process. The RGF uses phenomenological optical potentials, fitted to elastic proton-nucleus scattering. The loss of elastic flux into inelastic channels (either multinucleon knockout or non-nucleonic excitations) caused by the imaginary term of these potentials is recovered for the inclusive scattering, making use of dispersion relations. The larger cross section shown by the RGF indicates that the

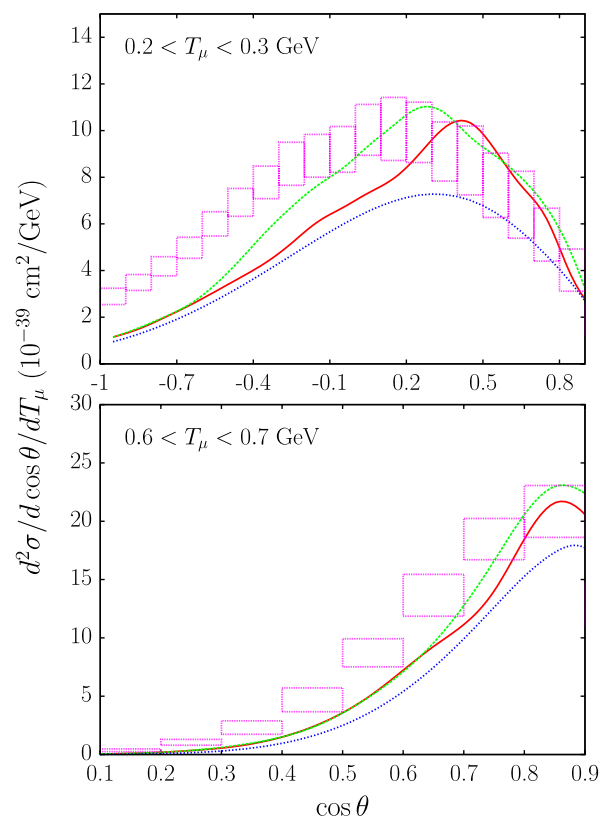

FIG. 2 (color online). Flux-averaged double-differential cross section per target nucleon for the $\mathrm{CCQE}{ }^{12} \mathrm{C}\left(\nu_{\mu}, \mu^{-}\right)$reaction displayed versus $\cos \theta$ for two bins of $T_{\mu}$. The results obtained with RMF [dotted dark gray (blue) line], RGF EDAD1 [solid (red) line], and RGF-EDAI [dotted light gray (green) line] potentials are compared with the MiniBooNE data of [1].

inelasticities represented in the phenomenological ROP $[16,26]$, when recovered by the RGF and included in the neutrino case, are more significant than for the inclusive electron scattering.

In Fig. 2, the flux-averaged double-differential cross sections are plotted versus $\cos \theta$ for two bins of $T_{\mu}$, i.e., $0.2<T_{\mu}<0.3 \mathrm{GeV}$ and $0.6<T_{\mu}<0.7 \mathrm{GeV}$. The approximate shape of the experimental cross section is well-described by the models. The RMF results generally underestimate the data, especially for the lower muon energy values; the agreement improves as $T_{\mu}$ increases. The RGF provides a better accordance with the size of the experimental cross section. The agreement is better for smaller angles, while the data are slightly underpredicted as $\theta$ increases. The RGF-EDAD1 yields, in general, a lower cross section than the RGF-EDAI, yet higher than the RMF one.

Finally, in Fig. 3, the total QE cross section per neutron obtained in the RMF and RGF models is displayed as a function of the neutrino energy $E_{\nu}$ and compared with the "unfolded" experimental data [1]. It was shown in [7] that the differences between the results of the RMF, superscaling, and rROP models tend to be washed out in the integration and that all these models, representing essentially the same nucleonic contribution to the inclusive cross sections, yield very similar results, all of them underpredicting the total MiniBooNE CCQE experimental cross section. Larger cross sections, in particular, for larger 


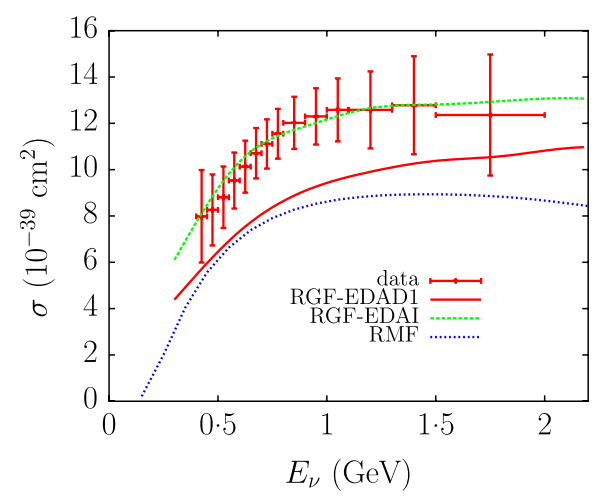

FIG. 3 (color online). Total CCQE cross section per neutron versus the neutrino energy. The cross sections calculated in the RMF [dotted dark gray (blue) line], RGF EDAD1 [solid (red) line], and RGF-EDAI [dotted light gray (green) line] potentials are compared with the flux unfolded MiniBooNE data of [1]

values of $E_{\nu}$, are obtained in the RGF with both optical potentials. The differences between RGF-EDAI and RGFEDAD1 are here clearly visible, RGF-EDAI being in good agreement with the shape and magnitude of the experimental cross section and RGF-EDAD1 being above RMF but clearly below the data. The differences between EDAI and EDAD1 are due to the different values of the imaginary parts of both potentials, particularly for the energies considered in kinematics with the lowest $\theta$ and the largest $T_{\mu}$. These kinematics, which were not considered in previous RGF calculations, give large contributions to the total cross section and emphasize the differences between the RGF predictions with both optical potentials. Notice that EDAI is a single-nucleus parameterization, which does have an edge in terms of better reproduction of the elastic proton $-{ }^{12} \mathrm{C}$ phenomenology [33] compared to EDAD1, and also leads to CCQE results in better agreement with data.

Summarizing, in this Letter, the results of the RMF and RGF models have been compared with the recent CCQE MiniBooNE data. Both models give a good description of the shape of the experimental cross sections. The RMF generally underpredicts the data, particularly for lower values of $\theta$ and $T_{\mu}$. In contrast, the RGF can give cross sections of the same magnitude as the experimental ones without the need to increase the standard value of the axial mass. The larger cross sections in the RGF model arise from the translation to the inclusive strength of the overall effect of inelastic channels. At present, lacking a phenomenological optical potential which exactly fulfills the dispersion relations in the whole energy region of interest, the RGF prediction is not univocally determined from the elastic phenomenology, although some preference to the EDAI predictions should be given.

Our results give a further and clear indication that, to clarify the content of the enhancement of the CCQE cross sections obtained in the RGF model compared to those of the IA models, a careful evaluation of all nuclear effects and of the relevance of multinucleon emission and of some non-nucleonic contributions [27] is required. This is important also to reconcile former results for which RMF was in good agreement with previous CCQE data [34]. A better determination of a phenomenological relativistic optical potential which closely fulfills the dispersion relations deserves further investigation.

This work was partially supported by DGI (Spain) under Contracts No. FIS2008-04189 and No. FPA2010-17142, the Spanish Consolider-Ingenio 2010 programme CPAN (CSD2007-00042), and by the INFN-CICYT collaboration agreements ACI2009-1053 and AIC10-D-000571.

[1] A. A. Aguilar-Arevalo et al. (MiniBooNE Collaboration), Phys. Rev. D 81, 092005 (2010).

[2] V. Lyubushkin et al., Eur. Phys. J. C 63, 355 (2009); P. Adamson et al., Phys. Rev. D 81, 072002 (2010).

[3] V. Bernard, L. Elouadrhiri, and U. G. Meissner, J. Phys. G 28, R1 (2002); A. Bodek et al., Eur. Phys. J. C 53, 349 (2008).

[4] O. Benhar, P. Coletti, and D. Meloni, Phys. Rev. Lett. 105, 132301 (2010); C. Juszczak, J. T. Sobczyk, and J. Zmuda, Phys. Rev. C 82, 045502 (2010).

[5] A. V. Butkevich, Phys. Rev. C 82, 055501 (2010).

[6] J.E. Amaro, M. B. Barbaro, J.A. Caballero, T.W. Donnelly, and C.F. Williamson, Phys. Lett. B 696, 151 (2011).

[7] J.E. Amaro, M.B. Barbaro, J. A. Caballero, T.W. Donnelly, and J.M. Udías, Phys. Rev. D 84, 033004 (2011).

[8] M. Martini, M. Ericson, G. Chanfray, and J. Marteau, Phys. Rev. C 80, 065501 (2009); 81, 045502 (2010).

[9] J. Nieves, I. Ruiz Simo, and M. J. Vicente Vacas, Phys. Rev. C 83, 045501 (2011); arXiv:1106.5374.

[10] A. De Pace, M. Nardi, W. M. Alberico, T. W. Donnelly, and A. Molinari, Nucl. Phys. A726, 303 (2003).

[11] S. Boffi, C. Giusti, F. D. Pacati, and M. Radici, Electromagnetic Response of Atomic Nuclei, Oxford Studies in Nuclear Physics Vol. 20 (Clarendon, Oxford, 1996); Phys. Rep. 226, 1 (1993).

[12] J. M. Udías, P. Sarriguren, E. Moya de Guerra, E. Garrido, and J. A. Caballero, Phys. Rev. C 48, 2731 (1993); 51, 3246 (1995).

[13] A. Meucci, C. Giusti, and F. D. Pacati, Phys. Rev. C 64, 014604 (2001).

[14] J. E. Amaro et al., Phys. Rev. C 71, 015501 (2005).

[15] A. Meucci, F. Capuzzi, C. Giusti, and F. D. Pacati, Phys. Rev. C 67, 054601 (2003).

[16] A. Meucci, J. A. Caballero, C. Giusti, F. D. Pacati, and J. M. Udías, Phys. Rev. C 80, 024605 (2009).

[17] J. A. Caballero, M.C. Martinez, J.L. Herraiz, and J. M. Udías, Phys. Lett. B 688, 250 (2010).

[18] C. Maieron, M. C. Martínez, J. A. Caballero, and J.M. Udías, Phys. Rev. C 68, 048501 (2003).

[19] J. A. Caballero, Phys. Rev. C 74, 015502 (2006).

[20] A. Meucci, C. Giusti, and F. D. Pacati, Nucl. Phys. A739, 277 (2004). 
[21] Y. Horikawa, F. Lenz, and N. C. Mukhopadhyay, Phys. Rev. C 22, 1680 (1980).

[22] J. A. Caballero, J.E. Amaro, M. B. Barbaro, T.W. Donnelly, and J.M. Udías, Phys. Lett. B 653, 366 (2007).

[23] F. Capuzzi, C. Giusti, and F. D. Pacati, Nucl. Phys. A524, 681 (1991).

[24] F. Capuzzi, C. Giusti, F. D. Pacati, and D. N. Kadrev, Ann. Phys. (N.Y.) 317, 492 (2005).

[25] A. Meucci, C. Giusti, and F. D. Pacati, Nucl. Phys. A756, 359 (2005).

[26] A. Meucci, J. A. Caballero, C. Giusti, and J. M. Udías, Phys. Rev. C 83, 064614 (2011).

[27] T. Leitner and U. Mosel, Phys. Rev. C 81, 064614 (2010).
[28] J.A. Caballero, J.E. Amaro, M.B. Barbaro, T.W. Donnelly, C. Maieron, and J.J.M. Udías, Phys. Rev. Lett. 95, 252502 (2005).

[29] J.E. Amaro, M. B. Barbaro, J. A. Caballero, and T.W. Donnelly, Phys. Rev. Lett. 98, 242501 (2007).

[30] A. Meucci, C. Giusti, and F. D. Pacati, Nucl. Phys. A773, 250 (2006).

[31] A. Meucci, C. Giusti, and F. D. Pacati, Acta Phys. Pol. B 37, 2279 (2006); 40, 2579 (2009).

[32] B. D. Serot and J. D. Walecka, Adv. Nucl. Phys. 16, 1 (1986); M. M. Sharma, M. A. Nagarajan, and P. Ring, Phys. Lett. B 312, 377 (1993).

[33] E. D. Cooper, S. Hama, B.C. Clark, and R. L. Mercer, Phys. Rev. C 47, 297 (1993).

[34] M. C. Martinez et al., Phys. Rev. C 73, 024607 (2006). 